\title{
On building models of spoken-word recognition: When there is as much to learn from natural "oddities" as artificial normality
}

\author{
Sven L. Mattys \\ University of Bristol, Bristol, England \\ AND \\ JULIE M. LISS \\ Arizona State University, Tempe, Arizona
}

\begin{abstract}
Much of what we know about spoken-word recognition comes from studies relying on speech stimuli either carefully produced in the laboratory or computer altered. Although such stimuli have allowed key constructs to be highlighted, the extent to which these constructs are operative in the processing of everyday speech is unclear. We argue that studying the recognition of naturally occurring degraded speech, such as that produced by individuals with neurological disease, can improve the external validity of existing spoken-word recognition models. This claim is illustrated in an experiment on the effect of talker-specific (indexical) variations on lexical access. We found that talker specificity effects, wherein words are better recalled if played in the same voice than in a different voice between two consecutive blocks, were greater when the words were spoken by dysarthric than by healthy individuals. The effects were found to relate to the increased processing time caused by the dysarthric stimuli, independently of their reduced intelligibility. This result is consistent with Luce, McLennan, and Charles-Luce's (2003) time-course hypothesis, which posits that reliance on indexical details increases when responses are delayed by suboptimal processing conditions. We conclude by advocating the use of laboratory and naturally occurring degraded speech in tandem and more systematic cross-talks between psycholinguistics and the speech sciences.
\end{abstract}

Speech scientists have long known that speech perfection is a fiction. Everyday speech is complex, variable, and, ultimately, elusive. Although attempts have been made to test spoken-word recognition models with everyday speech (e.g., Bard, Sotillo, Kelly, \& Aylett, 2001; Kemps, Ernestus, Schreuder, \& Baayen, 2004; McAllister, 1991; Mehta $\&$ Cutler, 1988), such studies are in a minority. There are two main reasons for this. First, studying conversational speech, by definition, limits the amount of experimental control one has over the input. Studying laboratory speech maximizes such control - for better or for worse. Second, attempting to define the concept of conversational speech itself opens a can of worms, because speech styles vary along quasiorthogonal dimensions, such as spontaneity (e.g., read, rehearsed, unscripted), articulatory effort (e.g., hyper-articulated, hypo-articulated), situation (e.g., monologue, dialogue), source (e.g., healthy speaker, dysarthric speaker), and so forth. These speech styles differ in the extent to which they contain the quantity and quality of acoustic cues available in carefully articulated laboratory speech, such as vowel reduction or phoneme elision/ assimilation (cf. Duez, 1995; Hawkins, 2003; Hawkins \& Smith, 2001; Tiffany, 1959; Uchanski, 2005), which makes the selection of stimuli for perceptual experiments rather arbitrary. For these reasons, the investigation of spoken-word recognition has traditionally relied on either carefully articulated speech produced in the laboratory or (re)synthesized speech.

\section{Artificial Normality}

Perceptual studies using tightly controlled laboratory speech have been successful in establishing key constructs for models of spoken-word recognition, such as lexical competition, bottom-up versus top-down flow, uniqueness point, and episodic versus abstract lexical representations. Yet the external validity of such constructs remains debatable. For instance, the finding that a word can be identified as soon as the sensory input makes it uniquely distinguishable from its competitors (see, e.g., misdem for misdemeanor) has been used to support the claim that lexical candidates are activated sequentially as the signal unfolds (e.g., Grosjean, 1980; Marslen-Wilson, 1987). However, Radeau, Morais, Mousty, and Bertelson (2000) found that sequential effects disappear when stimuli are played at average conversational speech rate. Likewise, Bard, Shillcock, and Altmann (1988) showed that, con-

S. L. Mattys, sven.mattys@bris.ac.uk 
trary to the sequential activation claim, people listening to conversational speech often need to hear substantial portions of the speech following the offset of a word in order to cope with its hypoarticulated characteristics.

Laboratory-generated phenomena reflect what the speech perception system can do with highly constrained input. The extent to which these capabilities play a role in processing the range of natural speech that listeners encounter every day is less clear. The field as a whole has not been insensitive to the merit of studying less idealized forms of speech, but a majority of degradation techniques have produced stimuli that, again, are unlikely to be found in everyday life because they defy realistic articulation (e.g., stimuli used in a majority of restoration, adaptation, and segmentation studies). Broader degradations such as sine-wave and noise-vocoded speech suffer from similar ecological limitations. In sum, everyday speech distortion rarely occurs in the narrow forms implemented in the laboratory. Instead, it tends to be complex and multidimensional, and it usually occurs in constellations. This is where validation based on laboratory research should be sought.

\section{Natural Oddities: What Can We Learn From the Perception of Naturally Occurring Degraded Speech?}

Nearly all of the speech that listeners encounter daily is degraded in acoustic quality and quantity relative to that spoken clearly in a quiet environment. In this article, we focus on the degradation that is endogenous to the speaker. We use the term naturally occurring degraded speech to refer to unedited speech stimuli produced by individuals who, for whatever reason, produce speech that is degraded relative to the speech produced by healthy, native speakers (e.g., dysarthric speech, deaf speech, stuttered speech).

Upon first thought, naturally occurring degraded speech may seem to be a rare occurrence, and the processes that it mobilizes may therefore seem qualitatively different from those engaged in the perception of normal speech. However, the National Institutes of Health estimate that approximately 46 million individuals in the United States suffer from communication disorders - roughly 1 in every 6 people (NIH, 2007). Dysarthria, in particular, is a key symptom of the most prevalent neurological disorders (Duffy, 2005). Likewise, Parkinson's disease alone affects approximately 1 million Americans (Iaconi, Zimmerman, Kulkarni, \& Balkrishnan, 2008), and it is estimated that roughly $90 \%$ of these people demonstrate degraded speech symptoms (Logeman, Fisher, Boshes, \& Blonsky, 1978). Exposure to endogenously degraded speech is, thus, far from uncommon.

Any successful model of speech processing must ultimately be able to accommodate the full range and variety of speech distortion that listeners encounter, and it must be able to explain how and when distortion interferes with speech processing. We take the position that naturally occurring degraded speech, even in severe forms, does not necessarily require or elicit different perceptual processing strategies than does healthy, native speech, but rather that the degradation presents a challenge and limits success for the typical strategies that listeners deploy (Liss, 2007).

Furthermore, many forms of naturally occurring degraded speech lend themselves to natural experiments (Bernstein \& Weismer, 2000) because their symptomatology often aligns with (i.e., selectively affects) theoretically relevant constructs. Table 1 provides a synopsis of the main characteristics associated with several common forms of speech production disorders. These characteristics constitute a useful testing ground for theoretical constructs (Table 2). Liss's work on the lexical segmentation of dysarthric speech provides an example of how naturally occurring degraded speech can be used to test existing models and illuminate issues that have not previously arisen with laboratory speech (Liss, Spitzer, Caviness, Adler, \& Edwards, 1998, 2000). Specifically, the various types of prosodic degradation found in dysarthria (Table 1) are of great relevance for models that have emphasized the role of stress for lexical segmentation (e.g., Cutler \& Norris, 1988). If the hypotheses generated from laboratory degraded speech are valid, they should apply to naturally occurring degraded speech that varies on theoretically relevant dimensions. This was the case in Liss et al.'s $(1998,2000)$ work. First, Liss et al. found that stress-based segmentation is robust: Listeners used stress to guide lexical segmentation even when key prosodic cues were attenuated by dysarthria. Second, some forms of prosodic degradation were more detrimental to stressbased segmentation than were others. This is of interest because, prior to these reports, studies evaluating differences in the application of stress-based strategies were largely cross-linguistic in focus, showing differences related to the rhythmic properties of the language (e.g., Vroomen \& de Gelder, 1995; Vroomen, Tuomainen, \& de Gelder, 1998). Liss et al.'s (1998, 2000) work showed that, for English speakers, the constellation of prosodic distortion in one type of dysarthric speech (hypokinetic) was less detrimental to the application of metrical segmentation than was that in another type (ataxic). Although the hypokinetic samples lacked in $f 0$ variation and were generally speeded, speech rhythm was largely preserved. The ataxic speech, in contrast, tended toward equal-and-even stress, a consequence of similar vowel durations in adjacent syllables. Thus, this comparative analysis of dysarthric speech allowed Liss et al. to highlight factors responsible for the success or failure of stress-based segmentation.

This observation led to a laboratory exercise using resynthesized unimpaired speech (Spitzer, Liss, \& Mattys, 2007), in which we examined the effects of reducing the primary acoustic cues of perceived syllabic stress $(f 0$, duration, and vowel quality). This was accomplished by flattening $f 0$ across each phrase, equalizing all syllable durations within each phrase, and adjusting first and second formant frequencies to coincide with neutral vowel (schwa) values. The results provided support that $f 0$ variations and the presence of fully realized vowels are important in ascertaining stress. Indeed, when these cues were minimized, the pattern of lexical boundary errors only weakly adhered to Cutler and Norris's (1988) stress-based 
Table 1

Main Articulatory and Perceptual/Acoustic Features Associated With Several of the More Common Forms of Speech Production Disorders

\begin{tabular}{|c|c|c|c|}
\hline Disorder & Articulatory Features & Prosodic Features & Voice and Resonance Features \\
\hline $\begin{array}{l}\text { Hypokinetic dysarthria } \\
\text { (Parkinsonian dysarthria) }\end{array}$ & $\begin{array}{l}\text { Imprecise articulatory contacts; } \\
\text { reduced vowel space }\end{array}$ & $\begin{array}{l}\text { Speech sounds rushed, little variation } \\
\text { in pitch, reduced speech loudness }\end{array}$ & $\begin{array}{l}\text { "Mumbled" sounding speech; weak } \\
\text { breathy voice with reduced loudness, } \\
\text { sometimes mild hypernasality }\end{array}$ \\
\hline Ataxic dysarthria & Irregular articulatory breakdown & $\begin{array}{l}\text { Slow speaking rate with a tendency } \\
\text { toward even syllabic stress; } \\
\text { "intoxicated" quality }\end{array}$ & Sometimes mild hypernasality \\
\hline Flaccid dysarthria & $\begin{array}{l}\text { If resulting from damage to } \\
\text { nerves innervating the face and } \\
\text { tongue: imprecise articulation } \\
\text { marked by regular phoneme } \\
\text { distortions, omissions, and } \\
\text { substitutions }\end{array}$ & $\begin{array}{l}\text { Generally, speaking rate is normal or } \\
\text { nearly so. Prosody is normal unless } \\
\text { there is laryngeal involvement, in } \\
\text { which case reduced pitch variation } \\
\text { may be noted }\end{array}$ & $\begin{array}{l}\text { If resulting from damage to the } \\
\text { laryngeal nerves: breathy voice, } \\
\text { reduced loudness, reduced pitch } \\
\text { variation. If resulting from damage to } \\
\text { the nerves of the velopharynx: } \\
\text { hypernasality, difficulty impounding } \\
\text { oral air pressure for stop consonants }\end{array}$ \\
\hline Spastic dysarthria & $\begin{array}{l}\text { Imprecise articulation } \\
\text { resulting in predominantly } \\
\text { phoneme distortions }\end{array}$ & $\begin{array}{l}\text { Slow, labored speaking rate, tendency } \\
\text { toward even syllabic stress; short } \\
\text { breath groups }\end{array}$ & $\begin{array}{l}\text { Strained-strangled vocal quality; } \\
\text { hypernasality }\end{array}$ \\
\hline $\begin{array}{l}\text { Abductor spasmodic } \\
\text { dysphonia }\end{array}$ & None & $\begin{array}{l}\text { Outward flow of speech halting, } \\
\text { compensatory speech rate slowing }\end{array}$ & $\begin{array}{l}\text { Strained-strangled voice quality; voice } \\
\text { stoppages }\end{array}$ \\
\hline Apraxia of speech & $\begin{array}{l}\text { Successive target approximation, } \\
\text { metathetic errors and syllable } \\
\text { transposition, perseverative and } \\
\text { anticipatory articulation errors }\end{array}$ & $\begin{array}{l}\text { Halting and labored progression of } \\
\text { speech, false starts, disruption or } \\
\text { flattening of prosodic contours }\end{array}$ & $\begin{array}{l}\text { Generally unimpaired, occasionally } \\
\text { hypernasal }\end{array}$ \\
\hline Stuttering & $\begin{array}{l}\text { Articulation is generally } \\
\text { controlled, however speech may } \\
\text { contain phoneme and syllable } \\
\text { repetitions, as well as phoneme } \\
\text { prolongations }\end{array}$ & $\begin{array}{l}\text { Problems in the outward flow of } \\
\text { speech, marked by blocks, } \\
\text { hesitations, sound prolongations and } \\
\text { repetitions; speaking rate } \\
\text { abnormalities may also be present }\end{array}$ & Generally unimpaired \\
\hline Deaf speech & $\begin{array}{l}\text { Imprecise segmental } \\
\text { articulation, especially vowels } \\
\text { (spectral confusion); shortened } \\
\text { frication and voicing intervals }\end{array}$ & $\begin{array}{l}\text { Rhythm and speech rate relatively } \\
\text { preserved; approximate sentential } \\
\text { prosody and poor intonation control }\end{array}$ & $\begin{array}{l}\text { Breathy voice quality; smaller range of } \\
\text { fundamental frequency; poor carrying } \\
\text { power }\end{array}$ \\
\hline
\end{tabular}

Note-Although each type of disorder has specific features, their manifestation is affected by severity, such that mild forms of each diagnostic category may sound quite different from severe forms (cf. Darley, Aronson, \& Brown, 1969).

segmentation hypothesis. Equalizing syllable duration had only a minimal effect.

The two studies described above-one with dysarthric speech, the other with resynthesized speech-converge in demonstrating the robustness of metrical segmentation in degraded conditions and further support the ecological validity of this process. Both also converge in demonstrating that some stress cues have a greater weight than others for segmentation. Of critical importance, however, is that the story told by the cue manipulations with resynthesized speech is insufficient to account for (or predict) the pattern of results elicited by the dysarthric speech. Specifically, the equalization of vowel durations, which might be considered the analogue to the equal-and-even duration disturbances in ataxic dysarthria, did not yield the expected decrements in lexical segmentation. Furthermore, the flattening of the $f 0$ was deleterious to the application of a metrical-stress-based strategy - an outcome not predicted by findings from the naturally occurring monotonous hypokinetic dysarthric speech. Thus, a greater insight into the mechanisms supporting natural speech recognition lies in the tandem exploration of natural and laboratory speech. Below, we further illustrate this point with a study showing how the mixed use of dysarthric and laboratory stimuli can improve our understanding of the effect of stimulus variability on spoken-word recognition.

\section{The Present Experiment}

Spoken words are better identified and recalled when they have been previously heard in the same voice than when they have been heard in a different voice (e.g., Mullennix, Pisoni, \& Martin, 1989; Pisoni, 1993) - that is, when the indexical characteristics of the signal (e.g., rate, pitch, voice quality) are preserved across presentations. Voice-specificity effects have been used to argue that lexical representations retain a certain amount of idiosyncrasy from individual tokens, making episodic traces relevant information rather than noise (Goldinger, 1996, 1998). However, these effects have also been varied as a function of tasks and stimulus quality. Luce, McLennan, and Charles-Luce (2003) argued that inconsistencies are best accounted for by differences in processing-time requirements (cf. McLennan \& Luce, 2005). According to this time-course hypothesis, the degree of reliance on instance-specific information is contingent on the speed with which a response is produced, with slow responses allowing retrieval of episodic traces to a greater extent than faster responses. 
Table 2

Examples of Possible Pairings Between Theoretical Constructs in Psycholinguistics and Speech Disorders Providing a Natural Testing Ground for Them

\begin{tabular}{|c|c|c|c|}
\hline Theoretical Construct & $\begin{array}{l}\text { Type of Disorder } \\
\text { or Form of Degradation }\end{array}$ & Key Properties & Hypothesis \\
\hline $\begin{array}{l}\text { Stress-based lexical } \\
\text { segmentation }\end{array}$ & $\begin{array}{l}\text { Various forms of dysarthria with } \\
\text { their characteristic decrements in } \\
\text { prosodic contrast }\end{array}$ & $\begin{array}{l}\text { Reduced syllabic strength } \\
\text { contrast }\end{array}$ & $\begin{array}{l}\text { Reduced syllabic strength contrasts increase } \\
\text { the incidence of lexical boundary errors; } \\
\text { differences in error patterns across dysarthria } \\
\text { forms and severities }\end{array}$ \\
\hline $\begin{array}{l}\text { Episodic traces in } \\
\text { lexical recognition }\end{array}$ & $\begin{array}{l}\text { Any disorder with reduced } \\
\text { intelligibility }\end{array}$ & $\begin{array}{l}\text { Diminished intelligibility, } \\
\text { perhaps with idiosyncratic } \\
\text { features }\end{array}$ & $\begin{array}{l}\text { Diminished intelligibility causes greater } \\
\text { reliance on episodic information }\end{array}$ \\
\hline $\begin{array}{l}\text { Lexical neighborhood } \\
\text { competition }\end{array}$ & $\begin{array}{l}\text { Any disorder whose intelligibility } \\
\text { deficit arises, at least in part, from } \\
\text { phonemic uncertainty (e.g., } \\
\text { flaccid dysarthria; deaf speech) }\end{array}$ & $\begin{array}{l}\text { Predictable phonemic } \\
\text { confusions }\end{array}$ & $\begin{array}{l}\text { Phonemic confusion alters lexical } \\
\text { competition in measurable ways }\end{array}$ \\
\hline $\begin{array}{l}\text { Word-initial cohort and } \\
\text { sequential activation }\end{array}$ & $\begin{array}{l}\text { Stuttered speech, speech with } \\
\text { disfluencies or filled pauses }\end{array}$ & $\begin{array}{l}\text { Word-onset enhancement due } \\
\text { to repetition or paralinguistic } \\
\text { intrusions }\end{array}$ & $\begin{array}{l}\text { Additional processing time allows } \\
\text { anticipatory lexical selection }\end{array}$ \\
\hline $\begin{array}{l}\text { Time-course of } \\
\text { lexical-semantic } \\
\text { integration }\end{array}$ & $\begin{array}{l}\text { Disorders with speech rate } \\
\text { consequences but minimal } \\
\text { coarticulatory disruption }\end{array}$ & $\begin{array}{l}\text { Altered speech rate with } \\
\text { minimal segmental or prosodic } \\
\text { disruption }\end{array}$ & $\begin{array}{l}\text { Context-driven lexical selection is modulated } \\
\text { by speech rate }\end{array}$ \\
\hline $\begin{array}{l}\text { Top-down effects on } \\
\text { perceptual learning }\end{array}$ & $\begin{array}{l}\text { Any disorder leading to reduced } \\
\text { intelligibility, accented speech }\end{array}$ & Reduced intelligibility & $\begin{array}{l}\text { Lexical knowledge promotes more robust } \\
\text { perceptual learning than does contextual } \\
\text { information }\end{array}$ \\
\hline Syntactic parsing & $\begin{array}{l}\text { Hypokinetic dysarthria with } \\
\text { reduced } f 0 \text { variation }\end{array}$ & $\begin{array}{l}\text { Deflated intonational/pitch } \\
\text { contour }\end{array}$ & $\begin{array}{l}\text { Sentential prosody assists syntactic parsing } \\
\text { and lexical segmentation }\end{array}$ \\
\hline
\end{tabular}

Dysarthric speech allows us to test this hypothesis in a straightforward way, since the degraded quality of dysarthric speech clearly puts the listener in suboptimal perceptual conditions. The following experiment compared voice-specificity effects in spoken words produced by healthy speakers and the same words produced by dysarthric individuals suffering from Huntington's disease (in a mild vs. severe form). The speech produced by the latter is categorized as hyperkinetic dysarthria, which is particularly well suited to a study of indexical specificity because of the generally peculiar speech pattern to which it gives rise and because of its idiosyncratic manifestation across productions (Darley, Aronson, \& Brown, 1969). Specificity effects were measured using a recall task similar to that used by Luce and Lyons (1998, Experiment 2). Unimpaired listeners were presented with two blocks of words produced by one of three types of speakers: (1) control speakers, (2) speakers with mild dysarthria, and (3) speakers with severe dysarthria. In each of the two blocks, some words were in a male voice; others, in a female voice. The listeners' task was to decide whether the words in Block 2 had been presented in Block 1, regardless of the speaker's voice. A voice-specificity effect should manifest itself as better recall for words heard in the same voice than for words heard in a different voice. More critically, Luce et al.'s (2003) time-course hypothesis predicts that this effect should be larger in dysarthric than in control stimuli, because the former are likely to occasion a notable processing-time delay.

\section{METHOD}

\section{Participants}

Seventy-two native speakers of American English received course credit or a small honorarium for taking part in the experiment. None reported a history of speech or hearing difficulties. Participants were randomly assigned to one of three speech types (control, mild dysarthria, severe dysarthria), with $n=24$ in each group.

\section{Materials and Design}

Eighty monosyllabic words were chosen, all of which were recorded by six speakers: A man and a woman with no known speech impairment (control), a man and a woman with mild dysarthria, and a man and a woman with severe dysarthria (see the next section for further details). Each participant heard only one speech type (control, mild, or severe). The experiment involved two successive blocks of 60 words each. None of the words were repeated within a block. Half the stimuli in each block were produced in the female voice and the other half in the male voice. The 60 words in Block 1 were the same for all participants, although the voice in which they were heard was counterbalanced across participants. In Block 2, 40 out of the 60 words heard in Block 1 were presented again, half in the same voice, half in the other voice. Following Luce and Lyons (1998), the same-voice stimuli in Block 2 were the same tokens as in Block 1. Which words in Block 2 were in the same or the different voice was counterbalanced across participants. The remaining 20 words in Block 2 had not been heard in Block 1. These were the same for all participants, with half of them in the male voice and half in the female voice.

\section{Speakers and Recording Procedure}

The words were recorded in the context of a larger investigation of the perception of dysarthric speech. Speech samples were collected in a sound-attenuated booth via a high-fidelity head-mounted microphone input to a laptop PC located outside the booth. The words were digitized at a $44.1-\mathrm{kHz}$ sampling rate using a custom acquisition software program (TF32; Milenkovic, 2004). The word files were then edited, and $50 \mathrm{msec}$ of silence was appended to the beginning and end of the words to avoid transition artifacts.

The four speakers with dysarthria were classified as mild or severe on the basis of the judgment of two certified speech-language pathologists. These professionals also confirmed that all four speakers with dysarthria exhibited patterns of speech consistent with a diagnosis of hyperkinetic dysarthria, resulting from intrusive, involuntary movements of the articulators and respiratory systems. 


\section{Testing Procedure}

Participants were told that the experiment consisted of two blocks of stimuli and that, in Block 1, they would hear a series of words and would simply have to pay attention to them. After each word, the participants pressed the space bar to play the next word. The task for Block 2 was not disclosed until the end of Block 1. For Block 2, participants were instructed to decide whether each word in Block 2 had been played in Block 1, ignoring voice differences. They gave their responses by pressing one of two keys labeled new and old. Both accuracy and speed were emphasized. To estimate the intelligibility of the words in the mild- and severe-impairment conditions, participants in those two conditions were administered a word transcription test at the end of Block 2. For that test, the words produced by the male and female speakers of the relevant impairment condition were randomized and played one at a time. Using a computer keyboard, the participants had up to $8 \mathrm{sec}$ to type in a transcription for each word.

\section{RESULTS}

\section{Main Patterns}

The word transcription data showed that average intelligibility in the mild and severe conditions was $62 \%$ and $26 \%$, respectively, which confirmed the appropriateness of the initial categorization. Response latencies to the words in Block 2 were measured from the onset of the spoken words. Only responses to words repeated across blocks (the old words) were analyzed. Incorrect responses were discarded. Because the distribution of latencies had a substantial positive skew (1.64), a logarithmic transformation was applied and latencies 2.5 standard deviations away from the mean were removed.

Taken together, the data showed a voice-specificity effect increasing with the level of impairment (see Figure 1). A generalized multilevel linear regression analysis run on the percentage of recall errors, with participants and items as random factors and impairment (control, mild, severe) and voice (same vs. different) as fixed factors, showed an effect of impairment $[F(2,2874)=7.21, p=.001]$, an effect of voice $[F(1,2874)=123.26, p<.001]$, and an interaction $[F(2,2874)=10.87, p<.001]$. Planned comparisons revealed a voice effect at all three levels of impairment [control, $F(1,958)=7.48, p<.01 ;$ mild, $F(1,958)=$ $55.10, p<.001$; severe, $F(1,958)=85.8, p<.001]$. A series of tests of $2 \times 2$ interactions showed that the voice effect in the control condition was smaller than that in the mild condition $[F(1,1916)=10.43, p<.005]$ and than that in the severe condition $[F(1,1916)=20.80, p<.001]$. The voice effect was not significantly different between the mild and severe conditions $[F(1,1916)=1.88, p=.17]$. The impairment effect resulted mainly from an accuracy decrement in the different-voice condition $[F(2,1437)=$ $17.00, p<.001]$. When the voice was the same, accuracy remained relatively high across impairment conditions $[F(2,1437)<1]$, which suggests that tolerance to degraded speech can be substantially counteracted by voice constancy, possibly because of similarity in fine acoustic detail and the absence of a need for voice normalization.

We ran a similar multilevel analysis on response latency, with word duration as a covariate to ensure that any difference between dysarthric and nondysarthric conditions could not simply be attributed to differences in speech rate. Word duration showed a main effect $[F(1,1727)=$ 66.46, $p<.001]$, but it did not interact with any of the main variables (all $F \mathrm{~s}<1$ ). Overall, the patterns were similar to those for accuracy [impairment, $F(2,1727)=$ $12.58, p<.001$; voice, $F(1,1727)=8.70, p<.005$; impairment $\times$ voice, $F(1,1727)=3.72, p<.05]$. Planned comparisons showed no voice effect in the control condition $[F(1,623)<1]$, but there was a significant voice effect in the mild condition $[F(1,561)=5.07, p<.05]$ and in the severe condition $[F(1,520)=9.87, p<.005] .{ }^{1} \mathrm{~A}$ series of $2 \times 2$ tests of interactions showed that the voice effect in the control condition was marginally smaller than that in the mild condition $[F(1,1184)=3.41, p=.06]$ and significantly smaller than that in the severe condition $[F(1,1143)=7.29, p<.01]$. The voice effect was not significantly different between the mild and severe conditions $[F(1,1082)<1]$. Contrary to the accuracy pattern, the impairment effect on latency was visible in both the same-voice and the different-voice conditions $[F(2,993)=6.97, p<.001$, and $F(2,718)=13.07, p<$ .001 , respectively]. Thus, recognition accuracy might not be affected by dysarthric degradation when the speaker's voice is the same, but the time that it takes to ascertain recognition is.

The time-course hypothesis was further confirmed in analyses performed separately on slow and fast respondents. Listeners were categorized as slow or fast within their impairment group on the basis of a median split of their average response latencies $(n=12$ for each subgroup). As can be seen in Figure 2, the voice effect pattern showed a marked contrast for the two subgroups. Whereas fast respondents showed no effect of voice $[F(1,901)<1]$ and no interaction between impairment and voice $[F(2,901)=1.11, p=.33]$, slow respondents showed both a voice effect $[F(1,798)=6.16, p=.01]$ and an impairment $\times$ voice interaction $[F(1,798)=3.97, p=$ $.02]$. The voice effect was present for both the mild condition $[F(1,250)=8.72, p<.005]$ and the severe condition

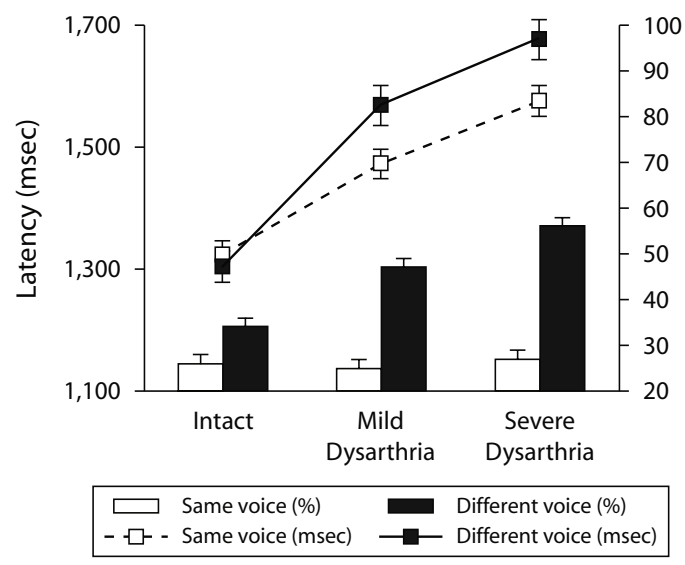

Figure 1. Correct recall latency (lines) and accuracy (bars) as a function of voice similarity and stimulus degradation due to dysarthria. Recall error \% is measured as $\mathbf{1 0 0}$ minus the percentage of correctly recalled words. 


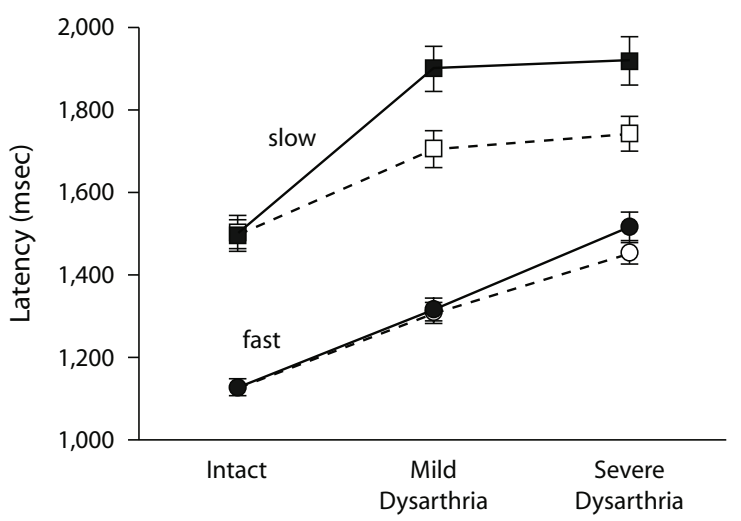

- $\square$ - - Same voice (slow) $\longrightarrow$ - Different voice (slow) - - O- - Same voice (fast) - Different voice (fast)

Figure 2. Correct recall latency for slow and fast respondents as a function of voice similarity and stimulus degradation due to dysarthria.

$[F(1,251)=4.52, p<.05]$. Thus, the increased reliance on voice specificity for the slower responses to dysarthric speech is consistent with Luce et al.'s (2003) time-course hypothesis: Under good processing conditions, when responses are fast, the retrieval of stimulus idiosyncrasies is minimal. When input quality is poor, and responses are slower, similarity in the surface characteristics of the stimuli proves a helpful aid to recalling.

\section{Dysarthria, Intelligibility, and Voice-Specificity Effects}

The well-documented reduced intelligibility of dysarthric speech (Yorkston, Strand, \& Kennedy, 1996) gives us the opportunity to tease apart the contributions of intelligibility and that of simple signal degradation to the recruitment of indexical details for lexical recognition. Specifically, the emergence of a voice effect at slow response latencies (i.e., the time-course hypothesis) could result not so much from longer processing time per se as from low intelligibility — of which longer latencies would only be a consequence. An explanation solely in terms of intelligibility must be ruled out, however. Indeed, the size of the voice effect, measured for each listener in the mild and severe groups $(n=48)$, did not correlate with the listeners' intelligibility scores $(r=-.14, p=.34)$, but it did with the listeners' average response latencies $(r=.42$, $p=.003)$. This correlation remained strong when intelligibility was controlled via partial correlation $(r=.40$, $p=.005)$. Thus, regardless of their intelligibility scores, the slower the respondents, the larger their voice effect.

In an attempt to partial out intelligibility more directly, we investigated the possibility that participants' poor performance in the different-voice dysarthric conditions resulted from their inability to identify the words in Block 2 as instances of the same words as those in Block 1. The words played in the same voice could have been responded to correctly via a simple acoustic match, bypassing lexical recognition. To test this possibility, we reanalyzed the performance of the participants in the mild and severe dysarthric groups, restricting our data set to the words correctly identified by these participants in the intelligibility test. Thus, latencies were measured only for the words in Block 2 that (1) were correctly identified in the intelligibility test - or showed identical transcriptions between the male and female voices - and (2) were correctly recalled in Block 2. Accuracy was calculated as the percentage of correctly recalled words among the correctly identified words. Despite the strict intelligibility criterion, an analysis run on the two dysarthric groups still showed a main voice effect [accuracy, $F(1,572)=15.78, p<.001$ (same voice, $M=26 \%$ recall error; different voice, $M=40 \%$ recall error); latency, $F(1,362)=8.25, p<.005$ (same voice, $M=1,458 \mathrm{msec}$; different voice, $M=1,569 \mathrm{msec})]$. The voice effect was not significantly modulated by group ( $F<1$ for both accuracy and latency). Thus, the emergence of a voice effect in the groups presented with dysarthric stimuli cannot be reduced to incomplete or incorrect encoding. Instead, listeners challenged by dysarthric speech called upon episodic details of words to a greater extent than did listeners processing undegraded stimuli.

\section{DISCUSSION}

The use of dysarthric speech alongside unimpaired speech allowed us to examine the effect of indexical variability on spoken-word recognition from a new perspective. Using naturally degraded stimuli, we provided confirmatory evidence for the ecological validity of Luce et al.'s (2003) time-course hypothesis without varying either the task or the content of the stimuli, and without artificial alterations to the stimuli. The data showed that the slower responses occasioned by processing dysarthric speech were accompanied with greater reliance on surface details of the stimuli. This pattern held even when intelligibility was controlled, which is particularly relevant to the time-course hypothesis, which posits time, rather than processing effort, as the cause of specificity effects, with activation confined to abstract representations in an early stage and spreading to episodic traces later on. The fact that intelligibility was not fundamentally at the root of the interaction between impairment (control vs. dysarthric) and voice-specificity effects also highlights the need to consider a range of qualitatively different speech styles when modeling psycholinguistic effects. One prediction from the present data is that speakers whose phonation or voice quality departs from average are likely to elicit response patterns similar to those observed in our dysarthric conditions.

This experiment not only has relevance for the ongoing debate about the interface between lexical access and the structure of the lexicon, but also illustrates how normal and naturally occurring degraded speech can be used in combination to address general questions about spokenword recognition. In this case, the use of dysarthric stimuli allowed us to put controlled, yet natural, pressure on the system and observe the coping mechanisms used by the listeners as a response. Thus, as in the experiments on speech segmentation described earlier (Liss et al., 1998, 
2000), naturally occurring degraded speech can act as a magnifying glass for patterns that would otherwise be difficult to highlight with laboratory speech or would require artificial distortions.

\section{CONCLUSION}

The goal of this article was to draw attention to and advocate the use of relatively unexplored speaker populations and empirical designs blending clinical and healthy speech for addressing fundamental questions about speech recognition. The merging of basic speech science and communication disorders has been promoted before: As Bernstein and Weismer (2000) have pointed out, "Work restricted to nonclinical populations can lull us into thinking that we know more than we do. Communication sciences can benefit from regarding data from disordered speakers and listeners as having the same value as data from normal speakers and listeners in the development of models and theories" (p. 231). On the basis of our own previous and present data, we have put this tenet into practice in the domain of spoken-word recognition. Specifically, our proposal is that naturally occurring degraded speech, and clinical speech disorders in particular, are a rich source of information for modeling normal speech recognition because naturally occurring degraded speech is encountered by listeners in everyday communication.

One could argue that naturally occurring degraded speech such as dysarthria has too many covarying features (i.e., extraneous to the primary variable of interest) to be of interpretive use. Dysarthric speech is indeed often accompanied by differences in delivery rate relative to healthy speech, and it can reveal atypical patterns at multiple levels simultaneously (e.g., subsegmental, segmental, and suprasegmental). However, the effects of such "confounded" factors and how they interact with the variables under study can in fact be profitably exploited in multidimensional analyses, which are notoriously effective at capturing the core components of natural data sets. Furthermore, many naturally occurring forms of degraded speech exhibit well-documented nonoverlapping constellations of articulatory features that have relevance for key theoretical constructs. Great interpretive power can be achieved by directly comparing contrastive degradations (e.g., hypokinetic vs. ataxic dysarthria; cf. Liss et al., 2000). More generally, however, we argue that the multidimensional nature of naturally occurring degraded speech is often a better reflection of the complexity of everyday healthy speech, or at least of the mechanisms engaged in the processing of healthy speech, than are many laboratory manipulations.

We do not claim that atypical speech should be approached as a proxy for conversational speech or that its experimental investigation surpasses or negates the benefits of that of laboratory speech. Rather, our claim is that the investigation of naturally occurring degraded speech coupled with mirror experimentation on laboratory speech can reveal some aspects of everyday speech processing that are difficult to measure with either approach in isolation. As illustrated in this article, the ve- ridical outliers afforded by dysarthrias are a rich testing ground for evaluating the capacities of the speech system under natural stress - a situation far from uncommon in conversational speech. By focusing research attention on the intersection between perceptual success and failure secondary to natural degradation, we magnify the details of typical perceptual processes. These details and the hypotheses that they generate can then be further explored in the laboratory. The tandem consideration of natural and laboratory speech can inform the ecological validity of speech-recognition models better than can the consideration of either speech type alone. Thus, naturally occurring degraded speech should be seen not as a special or unusual case of normal speech processing but, rather, as one of the many (natural) instances of speech that our processor is likely to encounter, and hence, as providing an opportunity for testing the robustness of current models and revealing phenomena that might not emerge as clearly within the highly controlled constraints of laboratory speech.

\section{AUTHOR NOTE}

This article was made possible by an International Scientific Interchange Scheme grant from the Biotechnology and Biological Sciences Research Council to S.L.M. (ISIS 1578) and a research grant from the National Institute on Deafness and Other Communicative Disorders, National Institutes of Health (5 R01 DC 6859) to J.M.L. We acknowledge the contributions of Kaitlin Lansford in data collection and analysis, as well as Mirjam Ernestus and Marc Brysbaert for their help with the statistical analyses. Correspondence can be sent to either S. L. Mattys, Department of Experimental Psychology, University of Bristol, 12A Priory Road, Bristol BS8 1TU, England (e-mail: sven.mattys@bris.ac.uk), or J. M. Liss, Motor Speech Disorders Laboratory, Department of Speech and Hearing Science, Arizona State University, Box 870102, Tempe, AZ 85281-0102 (e-mail: julie.liss@asu.edu).

\section{REFERENCES}

Bard, E. G., Shillcock, R. C., \& Altmann, G. T. M. (1988). The recognition of words after their acoustic offsets in spontaneous speech: Effects of subsequent context. Perception \& Psychophysics, 44, 395-408.

Bard, E. G., Sotillo, C., Kelly, M. L., \& Aylett, M. P. (2001). Taking the hit: Leaving some lexical competition to be resolved postlexically. Language \& Cognitive Processes, 16, 731-737.

Bernstein, L. E., \& Weismer, G. (2000). Basic science at the intersection of speech science and communication disorders. Journal of Phonetics, 28, 225-232.

Cutler, A., \& Norris, D. G. (1988). The role of stressed syllables in segmentation for lexical access. Journal of Experimental Psychology: Human Perception \& Performance, 14, 113-121.

Darley, F. L., Aronson, A. E., \& Brown, J. R. (1969). Differential diagnostic patterns of dysarthria. Journal of Speech \& Hearing Research, 12, 246-269.

Duez, D. (1995). On spontaneous French speech: Aspects of the reduction and contextual assimilation of voiced stops. Journal of Phonetics, 23, 407-427.

DufFy, J. (2005). Motor speech disorders: Substrates, differential diagnosis, and management (2nd ed.). St. Louis: Mosby.

GoLDINGER, S. D. (1996). Words and voices: Episodic traces in spoken word identification and recognition memory. Journal of Experimental Psychology: Learning, Memory, \& Cognition, 22, 1166-1183.

GoLDINGER, S. D. (1998). Echoes of echoes? An episodic theory of lexical access. Psychological Review, 105, 251-279.

Grosjean, F. (1980). Spoken word recognition processes and the gating paradigm. Perception \& Psychophysics, 28, 267-283.

Hawkins, S. (2003) Roles and representations of systematic fine 
phonetic detail in speech understanding. Journal of Phonetics, 31, 373-405.

Hawkins, S., \& SMith, R. (2001) Polysp: A polysystemic, phoneticallyrich approach to speech understanding. Italian Journal of Linguistics/ Rivista di Linguistica, 13, 99-188.

Iaconi, A. I., Zimmerman, M. A., Kulkarni, A. S., \& BalkrishNAN, R. (2008). Outcomes associated with pharmacologic treatments in Parkinson's disease: A review of recent literature. Expert Opinion on Pharmacotherapy, 9, 163-174.

Kemps, R., Ernestus, M. T. C., Schreuder, R., \& BaAyen, R. H (2004). Processing reduced word forms: The suffix restoration effect. Brain \& Language, 90, 117-127.

Liss, J. M. (2007). The role of speech perception in motor speech disorders. In G. Weismer (Ed.), Motor speech disorders (pp. 187-219). San Diego: Plural Publishing.

Liss, J. M., Spitzer, S., Caviness, J. N., Adler, C., \& Edwards, B. (1998). Syllabic strength and lexical boundary decisions in the perception of hypokinetic dysarthric speech. Journal of the Acoustical Society of America, 104, 2457-2466.

Liss, J. M., Spitzer, S., Caviness, J. N., Adler, C., \& Edwards, B. (2000). Lexical boundary error analysis in hypokinetic and ataxic dysarthria. Journal of the Acoustical Society of America, 107, 3415-3424.

Logeman, J. A., Fisher, H. B., Boshes, B., \& Blonsky, E. R. (1978) Frequency and cooccurrence of vocal tract dysfunctions in the speech of a large sample of Parkinson patients. Journal of Speech \& Hearing Disorders, 43, 47-57.

Luce, P. A., \& Lyons, E. A. (1998). Processing lexically embedded words. Journal of Experimental Psychology: Human Perception \& Performance, 25, 174-183.

Luce, P. A., McLennan, C. T., \& Charles-Luce, J. (2003). Abstractness and specificity in spoken word recognition: Indexical and allophonic variability in long-term repetition priming. In J. Bowers \& C. Marsolek (Eds.), Rethinking implicit memory (pp. 197-214). New York: Oxford University Press.

MarsLen-Wilson, W. D. (1987). Functional parallelism in spoken word-recognition. Cognition, 25, 71-102.

MCAllister, J. (1991). The processing of lexically stressed syllables in read and spontaneous speech. Language \& Speech, 34, 1-26.

McLennan, C. T., \& Luce, P. A. (2005). Examining the time course of indexical specificity effects in spoken word recognition. Journal of Experimental Psychology: Learning, Memory, \& Cognition, 31, 306-321.

Menta, G., \& Cutler, A. (1988). Detection of target phonemes in spontaneous and read speech. Language \& Speech, 31, 135-156.

Milenkovic, P. H. (2004). TF32 [Computer software]. Madison: University of Wisconsin, Department of Electrical and Computer Engineering.

Mullennix, J. W., Pisoni, D. B., \& Martin, C. (1989). Some effects of talker variability on spoken word recognition. Journal of the Acoustical Society of America, 85, 365-378.

National InStitutes of HeAlth (2007). National Institute of Deafness and Other Communication Disorders, Mission Statement (Sep- tember 21, 2007). Retrieved January 20, 2008, from www.nidcd.nih .gov/about/learn/mission.asp

Pisoni, D. B. (1993). Long-term memory in speech perception: Some new findings on talker variability, speaking rate, and perceptual learning. Speech Communication, 13, 109-125.

Radeau, M., Morais, J., Mousty, P., \& Bertelson, P. (2000). The effect of speaking rate on the role of the uniqueness point in spoken word recognition. Journal of Memory \& Language, 42, 406-422.

Spitzer, S. M., Liss, J. M., \& MAtTys, S. L. (2007). Acoustic cues to lexical segmentation: A study of resynthesized speech. Journal of the Acoustical Society of America, 122, 3678-3687.

TIFFANY, W. R. (1959). Nonrandom sources of variation in vowel quality. Journal of Speech \& Hearing Research, 2, 305-317.

UCHANSKI, R. M. (2005). Clear speech. In D. B. Pisoni \& R. E. Remez, (Eds.), The handbook of speech perception (pp. 207-235). Oxford: Blackwell.

VRoomen, J., \& de Gelder, B. (1995). Metrical segmentation and lexical inhibition in spoken word recognition. Journal of Experimental Psychology: Human Perception \& Performance, 21, 98-108.

Vroomen, J., Tuomainen, J., \& DE Gelder, B. (1998). The roles of word stress and vowel harmony in speech segmentation. Journal of Memory \& Language, 38, 133-149.

Yorkston, K. M., Strand, E. A., \& Kennedy, M. R. (1996). Comprehensibility of dysarthric speech: Implications for assessment and treatment planning. American Journal of Speech-Language Pathology, 5, 55-66.

\section{NOTE}

1. Luce and Lyons (1998), using a similar design with intact stimuli only, found a voice effect on latency but not on accuracy, a pattern opposite to ours. This could have resulted from slight differences in design between the two studies. In our experiment, the passive listening feature in Block 1, which was imposed by the reduced intelligibility of the impaired stimuli and the unavailability of nonwords from our dysarthric database, contrasted with the lexical decision task used by Luce and Lyons. Thus, in the Luce and Lyons study, the lexical decision task forced participants to pay attention explicitly to the lexical status of the stimuli. Moreover, because of the lexical-decision task, the total number of real words to encode in Luce and Lyons' Block 1 was half that in our study (30 vs. 60); so was the number of old words to recall in Block 2 (20 vs. 40). Therefore, the absence of a voice effect on latency in our intact group could have resulted from somewhat shallower encoding during Block 1. As for recall accuracy, Luce and Lyons's participants were near ceiling, which could have made a voice effect difficult to obtain. Our lower accuracy, a consequence of reduced encoding, would not have suffered from this ceiling effect. However, because the main issue for the present study is the change in voice effect across impairment conditions, whether the voice effect arises in terms of latency or accuracy is not of primary concern.

(Manuscript received March 21, 2007, revision accepted for publication March 25, 2008.) 